\title{
PLGA-PEG-PLGA hydrogel with NEP1-40 promotes the functional recovery of brachial plexus root avulsion in adult rats
}

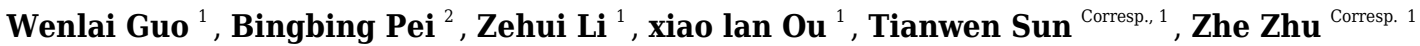 \\ 1 Department of Hand Surgery, the Second Hospital of Jilin University, Chang chun, Jilin, China \\ 2 Department of Orthopedics, Chinese People's Liberation Army Joint Logistics, Support Unit 964 Hospital, Chang chun, Jilin, China \\ Corresponding Authors: Tianwen Sun, Zhe Zhu \\ Email address: suntianwen@jlu.edu.cn, zhuzhe1983@jlu.edu.cn
}

Adult brachial plexus root avulsion can cause serious damage to nerve tissue and impair axonal regeneration, making the recovery of nerve function difficult. Nogo-A extracellular peptide residues 1-40 (NEP1-40) promote axonal regeneration by inhibiting the Nogo-66 receptor (NgR1), and poly (D, L-lactide-co-glycolide)-poly (ethylene glycol)-poly (D, Llactide-co-glycolide) (PLGA-PEG-PLGA) hydrogel can be used to fill in tissue defects and concurrently function to sustain the release of NEP1-40. In this study, we established an adult rat model of brachial plexus nerve root avulsion injury and conducted nerve root replantation. PLGA-PEG-PLGA hydrogel combined with NEP1-40 was used to promote nerve regeneration and functional recovery in this rat model. Our results demonstrated that functional recovery was enhanced, and the survival rate of spinal anterior horn motoneurons was higher in rats that received a combination of PLGA-PEG-PLGA hydrogel and NEP1-40 than in those receiving other treatments. The combined therapy also significantly increased the number of fluorescent retrogradely labeledneurons, muscle fiber diameter, and motor endplate area of the biceps brachii. In conclusion, this study demonstrates that the effects of PLGA-PEG-PLGA hydrogel combined with NEP1-40 are superior to those of other therapies used to treat brachial plexus nerve root avulsion injury. Therefore, future studies should investigate the potential of PLGA-PEG-PLGA hydrogel as a primary treatment for brachial plexus root avulsion. 
1 PLGA-PEG-PLGA hydrogel with NEP1-40 promotes the functional recovery of brachial plexus root avulsion in adult rats

4 Wenlai Guo ${ }^{1}$, Bingbing Pei ${ }^{2}$, Zehui $\mathrm{Li}^{1}$, Xiaolan $\mathrm{Ou}^{1}$, Tianwen Sun ${ }^{1 *}$, Zhe Zhu ${ }^{1 *}$

$6{ }^{1}$ Department of Hand Surgery, the Second Hospital of Jilin University, 218 Lane Ziqiang, 7 Nanguan District, Changchun, Jilin, 130041, P. R. China.

$8 \quad 2$ Department of Orthopedics, Chinese People's Liberation Army Joint Logistics, Support Unit 9964 Hospital, Jilin Province, Changchun, Xi’ an Street, No.4799, P. R. China.

*Correspondence should be addressed to: Tianwen Sun, suntianwen@jlu.edu.cn; Zhe Zhu, zhuzhe1983@jlu.edu.cn

Department of Hand Surgery, the Second Hospital of Jilin University, 218 Lane Ziqiang, Nanguan District, Changchun, Jilin, 130041, P. R. China.

\section{Abstract}

Adult brachial plexus root avulsion can cause serious damage to nerve tissue and impair axonal regeneration, making the recovery of nerve function difficult. Nogo-A extracellular peptide residues 1-40 (NEP1-40) promote axonal regeneration by inhibiting the Nogo-66 receptor (NgR1), and poly (D, L-lactide-co-glycolide)-poly (ethylene glycol)-poly (D, L-lactideco-glycolide) (PLGA-PEG-PLGA) hydrogel can be used to fill in tissue defects and concurrently function to sustain the release of NEP1-40. In this study, we established an adult rat model of brachial plexus nerve root avulsion injury and conducted nerve root replantation. PLGA-PEGPLGA hydrogel combined with NEP1-40 was used to promote nerve regeneration and functional recovery in this rat model. Our results demonstrated that functional recovery was enhanced, and the survival rate of spinal anterior horn motoneurons was higher in rats that received a combination of PLGA-PEG-PLGA hydrogel and NEP1-40 than in those receiving other 
treatments. The combined therapy also significantly increased the number of fluorescent retrogradely labeled neurons, muscle fiber diameter, and motor endplate area of the biceps brachii. In conclusion, this study demonstrates that the effects of PLGA-PEG-PLGA hydrogel combined with NEP1-40 are superior to those of other therapies used to treat brachial plexus nerve root avulsion injury. Therefore, future studies should investigate the potential of PLGAPEG-PLGA hydrogel as a primary treatment for brachial plexus root avulsion.

Keywords: PLGA-PEG-PLGA, brachial plexus avulsion, NEP1-40, nerve regeneration

\section{Introduction}

Brachial plexus avulsion (BPA) is a commonly observed injury in young adults and is often caused by severe trauma, such as that associated with car accidents (Faglioni et al. 2014). After BPA, the nerve root is torn from the spinal cord, which causes loss of sensory and motor functions in the corresponding area of innervation. The initial insult and secondary damage result in widespread neuronal death. Unfortunately, nerve regeneration is difficult because of various inhibitory factors, and recovery is almost impossible without surgical treatment. Further, simple surgical treatment often leads to unsatisfactory results that are accompanied by a high disability rate (Carlstedt 2008; Gibon et al. 2016; Lang et al. 2005).

Replantation of the avulsed nerve root in situ is the ideal treatment for BPA. Replantation can not only restore the anatomical structure and avoid injuries caused by other reconstructive interventions (Eggers et al. 2010; Limthongthang et al. 2013; Shin et al. 2005), but also greatly reduces post-injury neuralgia (Ciaramitaro et al. 2017; Teixeira et al. 2015; Zhou et al. 2017). Nevertheless, various inhibitory factors produced in post-injury nerve tissue, limit neural regeneration and result in poor functional recovery (Kwok-FaiSo \& Xiao-MingXu 2015). To promote the recovery of nerve function, more attention has been given to the promotion of axon regeneration or the elimination of inhibitory factors associated with nerve damage.

Nogo receptors (NgRs), including $\mathrm{NgR} 1, \mathrm{NgR} 2$, and $\mathrm{NgR} 3$, are endogenous myelin- 
associated protein-related receptors in the central and peripheral nerves (Chen et al. 2000; Young 1996). Nogo-A, the ligand with the highest affinity for $\mathrm{NgR} 1$, can bind to $\mathrm{NgR} 1$, inhibit axonal growth, resulting in growth cone collapse. Nogo-A extracellular peptide residues 1-40 (NEP140), the most common $\mathrm{NgR} 1$ antagonist, comprises a small polypeptide that competes with NgR1 by targeting Nogo-66, the main inhibitory region of Nogo-A (GrandPré et al. 2002). Therefore, NEP1-40 can relieve the neurosuppressive effect of Nogo-A (Cao et al. 2008; Chen et al. 2000; GrandPré et al. 2002; Kempf \& Schwab 2013; Xu et al. 2017b), increase the expression of growth-associated protein 43 (GAP-43) and microtubule-associated protein 2 (MAP-2) in nerve tissue, reduce the levels of amyloid- $\beta$ (A4) precursor protein (APP), and, consequently, facilitate nerve regeneration, axonal growth as well as functional recovery (Li \& Strittmatter 2003; Mingorance et al. 2006; Wang et al. 2007; Xu et al. 2017b).

BPA can (i) cause nerve tissue defects, lead to an irregular cavity at the junction area between the spinal cord and the brachial plexus, (ii) subsequently inducing the excessive buildup of scar tissue, and, (iii) finally, impact the penetration of axons (Carlstedt 2008; Meng et al. 2019; Meng et al. 2020). Therefore, the use of appropriate filling materials with a drug load may be employed to promote nerve regeneration (Baazil et al. 2015; Ding et al. 2019). Poly (D, Llactide-co-glycolide)-poly (ethylene glycol)-poly (D, L-lactide-co-glycolide) (PLGA-PEGPLGA) is a temperature-sensitive hydrogel that has a wide range of applications in drug delivery owing to its good biocompatibility and safety. The fluidity and gel-forming properties of PLGAPEG-PLGA allow it to effectively fill in tissue defects and sustain the delivery of drugs to the site of injury. (Ding et al. 2019; Zhang et al. 2014a) Further, studies have reported that combinations of PLGA-PEG-PLGA hydrogel with drugs can be more efficacious than drugs alone (Ding et al. 2019; Huang et al. 2020). Because treatment with drugs alone, if it is intravenous injection, after the metabolism of the various organs of the body, the actual dose of the drug reaching the injured site is very small, and it cannot even meet the needs of the injured site for treatment. Simply increasing the injected dose will not only affect the function of the other organs, and more drugs are wasted. If the drug is injected directly in situ, the fluidity of the 
82

drug liquid and the irregular shape of the injured site will cause the loss of most drugs and the utilization rate is low. But the drug-loaded hydrogel can provide excellent encapsulation for NEP1-40 and avoid sudden release of it. Therefore, it will increase the efficacy of the drug (Macaya \& Spector 2012; Shrestha et al. 2014; Zhang et al. 2011). Therefore, it can facilitate nerve regeneration. The current study aimed to examine the effects of PLGA-PEG-PLGA hydrogel loaded with NEP1-40 on neural regeneration in an adult rat model of BPA and evaluate the mechanisms underlying regeneration.

\section{Materials \& Methods}

\subsection{Materials}

PEG (Mn = 1500 g/mol), D, L-Lactide (D, L-LA), glycolide (GA), Tin (II) 2-ethylhexanoate $\left(\mathrm{Sn}(\mathrm{Oct})_{2}\right)$, methyl thiazolyl tetrazolium, and quercetin were purchased from Sigma-Aldrich (Sigma, St Louis. MO, USA). NEP1-40 was purchased from TOCRIS (Bristol, UK). Sheep antirat choline acetyltransferase (ChAT) antibody was purchased from Millipore (Darmstadt, Germany), while rabbit anti-goat fluorescence 568 antibody and alpha-bungarotoxin ( $\alpha$-BTX) 594 antibody were purchased from Abcam (Cambridge, UK) and Invitrogen (California, USA), respectively. Fluoro-Gold (FG) was purchased from Denver (Colorado, USA). All chemical reagents were used directly without further purification.

\subsection{Synthesis of the temperature-sensitive PLGA-PEG-PLGA hydrogel}

As described previously (Ci et al. 2014; Zhao et al. 2014), firstly, $68 \mathrm{~g}$ of PEG, $115 \mathrm{~g}$ of D, L-LA, and $35 \mathrm{~g}$ of GA were dehydrated by vacuum drying for $48 \mathrm{~h}$. Subsequently, $30 \mathrm{mg}$ of Sn $(\mathrm{Oct})_{2}$ was added as a catalyst at $120{ }^{\circ} \mathrm{C}$, and the reaction was allowed to continue for $48 \mathrm{~h}$. The reaction product was sufficiently dissolved in $500 \mathrm{ml}$ cold water at $4{ }^{\circ} \mathrm{C}$, and the solution was heated to $80{ }^{\circ} \mathrm{C}$ for precipitation and purification. The purification process was repeated thrice, and the purified product was dried in a vacuum for later use.

\subsection{Characterization of PLGA-PEG-PLGA}

Experimental data were recorded using a Bruker DMX500 spectrometer (Switzerland) at 
$500 \mathrm{MHz}$ Deuterated chloroform $\left(\mathrm{CDCl}_{3}\right)$ was used as a solvent, and tetramethylsilane was used as an internal standard. The sol-gel transition temperature of the PLGA-PEG-PLGA triblock copolymer was determined by the vial inversion test. Different quantities of PLGA-PEG-PLGA polymer were dissolved in phosphate-buffered saline (PBS), and the vial containing $0.5 \mathrm{~mL}$ of the polymer solution was stored for 15 min prior to the measurement. The temperature increment was set at $1{ }^{\circ} \mathrm{C}$. The gel was successfully formed when the solution in the vial did not flow after being inverted for $30 \mathrm{~s}$.

\subsection{Release profiles of NEP1-40 from PLGA-PEG-PLGA hydrogels}

As described above, the optimal mass concentration of the PLGA-PEG-PLGA polymer was prepared. Then, NEP1-40 was dissolved in acetone and added to the PLGA-PEG-PLGA solution. Subsequently, the PLGA-PEG-PLGA polymer loaded with NEP1-40 was added to a test tube with an inner diameter of $16 \mathrm{~mm}$ and incubated at $37{ }^{\circ} \mathrm{C}$ for $10 \mathrm{~min}$. After the hydrogels were

formed, $5 \mathrm{~mL}$ of PBS were added to the top of the gels, and the test tubes were placed at $37{ }^{\circ} \mathrm{C}$ with continuous shaking at $60 \mathrm{rpm}$. Subsequently, $1 \mathrm{~mL}$ of PBS was regularly aspirated and replenished every 2 days, with a total time of 14 days (Nakamura et al. 2011b; Xu et al. 2017b; Zhai \& Feng 2019). The amount of NEP1-40 in the aspirated PBS extract was measured using an ultraviolet spectrophotometer at $306 \mathrm{~nm}$, and drug release profile was constructed on the basis of the obtained absorbance values.

\subsection{Experimental animals and groups}

Animal procedures were conducted in accordance with the guidelines for the review and approval of the Animal Care and Use Committee of Jilin University (Ethical Approval No.2018119), and followed the Guide for the Care and Use of Laboratory Animals issued by the USA National Institutes of Health (National \& Promising 2009). Effort was made to minimize pain and the number of animals used in the experiments. The dose used for anesthesia is $45 \mathrm{mg} / \mathrm{kg}$ of 3\% pentobarbital sodium (New Asia Pharmaceutical, Shanghai, China), and the dose used for euthanasia is $200 \mathrm{mg} / \mathrm{kg}$ of $3 \%$ pentobarbital sodium.

Forty healthy female adult Sprague-Dawley rats (weight, 200-250 g; age, 10-12 weeks) were 
provided by the Animal Experimental Center of Jilin University in China. The reasons why we choose female adult rats are as following: (1) female rats are less aggressive and have no sense of territory, so they are less aggressive to each other; while male rats have territorial awareness, they may attack each other, causing the experiment to fail; (2) after brachial plexus injury, some rats will be aggressive or eat limbs that do not feel sensation due to loss of sensation on one side. Females are less aggressive, so they are less likely to eat their limbs. All animals were caged in standard animal rooms at $22{ }^{\circ} \mathrm{C}$ with free access to food and water. The rats were randomized into the following four groups ( $\mathrm{n}=10$ /group): control group, blank hydrogel group, NEP1-40 group, and NEP1-40-loaded hydrogel group. Experienced technicians for health and behavior monitored the animals daily during the protocol. The animals were weighed weekly. No rats displayed markers associated with death or poor prognosis of quality of life, or specific signs of severe suffering or distress, which would have led to early and immediate euthanasia.

\subsection{Model preparation and treatments}

The rats were intraperitoneally anesthetized using $45 \mathrm{mg} / \mathrm{kg}$ of $3 \%$ pentobarbital sodium. The BPA model was established according to the methods described by Gu et al. (Gu et al. 2004). Briefly, the backs of rats were depilated, and the lamina and spinous processes from the fourth cervical spine (C4) to the second thoracic spine (T2) were exposed via the dorsal approach. The dorsal lamina of the right $\mathrm{C} 5-\mathrm{C} 7$ was opened followed by exposure and avulsion of the C5-C7 nerve roots. The $\mathrm{C} 5$ and $\mathrm{C} 7$ nerve roots (about $5 \mathrm{~mm}$ in length) were excised, and the distal ends of the nerve roots were retracted to prevent regeneration. The $\mathrm{C} 6$ nerve root was replanted to the spinal cord, and the muscles and skin were sutured after hemostasis. After surgery, the animals were maintained in a clean environment and administered an intramuscular injection of cefazolin sodium (50 mg/kg, twice a day; Zhong-nuo Pharmaceutical) as anti-infection treatment for 3 consecutive days (Birrell \& Fuller 2019; Tetsuro \& Kusaba 2009).

In the control group, the surgical incision was sutured directly, and a $100 \mu \mathrm{L}$ mixture of PBS and dimethyl sulfoxide ((DMSO) $(83 \%$ PBS + 17\% DMSO) was administered intraperitoneally once a day for 14 consecutive days (Table 1). In the blank hydrogel group, $100 \mu \mathrm{L}$ of hydrogel 
was injected around the replanted C6 nerve root and held for $3 \mathrm{~min}$. The incision was sutured until the hydrogel was stabilized. Postoperatively, a $100 \mu \mathrm{L}$ mixture of PBS and DMSO (83\% PBS $+17 \%$ DMSO) was intraperitoneally administered once a day for 14 consecutive days (Table 1). In the NEP1-40 group, the surgical incision was directly sutured, and $12.5 \mu \mathrm{g}$ of NEP1-40 were dissolved in a $100 \mu \mathrm{L}$ mixture of PBS and DMSO (83\% PBS + 17\% DMSO) and intraperitoneally injected once a day for 14 consecutive days (Table 1) (Nakamura et al. 2011b; Steward et al. 2008; $\mathrm{Xu}$ et al. 2017b). In the NEP1-40-loaded hydrogel group, $100 \mu \mathrm{L}$ of 0.5 mg/mL NEP1-40-loaded hydrogel was injected around the replanted C6 nerve root and allowed to stabilize for $3 \mathrm{~min}$. The incision was then sutured. Postoperatively, a $100 \mu \mathrm{L}$ mixture of PBS and DMSO (83\% PBS + 17\% DMSO) was intraperitoneally administered once a day for 14 consecutive days (Table 1).

\subsection{Behavioural test}

Between weeks 2 and 6 post-surgery, eight rats from each group were subjected to the Terzis grooming test (TGT) test to evaluate motor function of the affected upper limb (Bertelli \& Mira 1993b; Kwok-FaiSo \& Xiao-MingXu 2015). Two investigators who did not participate in model induction graded the motor function of the right upper limb. Divergences between assessments of the two investigators were independently resolved by a third investigator.

\subsection{Immunofluorescence assay and FG nerve retrograde labeling}

At 6 weeks post operation, three rats from each group were randomly selected and sacrificed. After infusion with 4\% paraformaldehyde, the spinal C6 segment was dissected and cut into 25$\mu \mathrm{m}$ thick sections. One out of every four sections was incubated with the anti-ChAT (1:100; Millipore) antibody at $4{ }^{\circ} \mathrm{C}$ overnight. The following day, sections were incubated with the secondary antibody at $18-22{ }^{\circ} \mathrm{C}$ for $1.5 \mathrm{~h}$ (Alexa Fluor ${ }^{\circledR} 568$ rabbit anti-goat IgG, Abcam). ChAT-positive spinal anterior horn motoneurons on the injured side and contralateral side were counted under a fluorescence microscope to assess the cell ratio of the injured side to the healthy side.

At 6 weeks post operation, three rats were randomly selected from each group. They were 
anesthetized and a transverse incision was made below the right clavicle. The pectoralis major and minor muscles were cut off to expose and dissociate the musculocutaneous nerve of the right side. A glass needle was inserted $5 \mathrm{~mm}$ proximal to the nerve entry point of the musculocutaneous nerve into the biceps muscle, and $0.8 \mu \mathrm{L}$ of FG was carefully injected into the musculocutaneous nerve with a microinjection pump. The needle was retained for $10 \mathrm{~s}$ until the FG was completely absorbed. The muscle and skin were sutured layer by layer. Postoperative anti-infection treatment was administered for 3 consecutive days. Subsequently, the rats were subjected to cardiac perfusion using $4 \%$ paraformaldehyde, and the $\mathrm{C} 5-\mathrm{C} 8$ segments were longitudinally cut into $25-\mu \mathrm{m}$ thick sections. One out of every four sections was observed under a fluorescence microscope to assess the number of FG-labeled neurons.

\subsection{Immunofluorescence detection of muscle fiber diameter in the injured biceps and} assessment of the amount and morphology of motor endplate (MEP) neurons in the injured biceps muscle by immunofluorescence

At 6 weeks post operation, four rats were randomly selected and sacrificed from each group and subjected to cardiac perfusion using $4 \%$ paraformaldehyde. The biceps muscles on the injured side were dissected, cut into $14-\mu \mathrm{m}$ thick sections, and stained with hematoxylin-eosin. We randomly selected and photographed 50-100 muscle fibers from each biceps muscle under a light microscope. Image-J (National Institutes of Health, Bethesda, MD, USA) was used to measure the diameter of the muscle fiber, and the average value was calculated.

One out of every four sections was used to stain the MEP. The sections were incubated with $\alpha$-BTX 594 antibody in $0.01 \mathrm{M}$ PBS ( $\alpha$-BTX 594: 0.01M PBS=1:400) for $30 \mathrm{~min}$, and the morphology of the MEPs was observed under a microscope. At least 150 MEPs were randomly selected from each animal specimen and photographed. Image-J analysis software was used to measure the area of the MEPs.

\subsection{Statistical analyses}

Graph-Pad Prism 5.0 cartography software (Graph-Pad Software, Inc., CA, USA) was used for mapping, and SPSS 15.0 software (SPSS, Chicago, IL, USA) was used for one-way analysis 
217 of variance. Measurement data are expressed as means \pm standard deviation (SD). A pairwise 218 comparison was performed using the Students $t$-test. Differences with a P-value of $\leq 0.05$ were 219 considered statistically significant.

220

221

222

\section{Results and discussion}

\subsection{Material characterization and release profile of NEP1-40}

PLGA-PEG-PLGA temperature-sensitive hydrogel can (i) fill defected tissues, (ii) restrict the ingrowth of some cell types, including fibroblasts and inflammatory cells, and (iii) prevent astrocyte proliferation, thereby minimizing the formation of scar tissue. New tissues will completely fill the injured area following the absorbance, degradation, and disappearance of the scaffold (Luo et al. 2004; Rong et al. 2019; Zhang et al. 2014b). In this study, we first synthesized PLGA-PEG-PLGA hydrogels and determined their properties.

The composition and structure of the PLGA-PEG-PLGA copolymer were determined using ${ }^{1} \mathrm{H}$ NMR spectroscopy (Figure 1). We observed a complicated split in these peaks owing to the random copolymerization of GA and LA, and the characteristic signals that appeared at 5.2, 4.8, 3.5, and $1.5 \mathrm{ppm}$ represented the $\mathrm{CH}$ of $\mathrm{LA}, \mathrm{CH}_{2}$ of $\mathrm{GA}, \mathrm{CH}_{2}$ of $\mathrm{PEG}$, and $\mathrm{CH}_{3}$ of $\mathrm{LA}$, respectively. Studies have revealed that there is a close association between the sol-gel transition of PLGA-PEG-PLGA hydrogel and its concentration (Zhang et al. 2011). In this study, the vial inversion test was performed to explore the sol-gel transition of the hydrogels (Figure 2), and a typical sol-gel-precipitation transition was observed when the hydrogel concentration was 15$30 \%$. Our findings revealed that a higher PLGA-PEG-PLGA concentration was associated with a higher gel-precipitation transition temperature and a lower sol-gel transition temperature, and a 30wt\% PLGA-PEG-PLGA hydrogel had a wider gel window. Therefore, the $30 \mathrm{wt} \%$ PLGAPEG-PLGA hydrogel was selected for later use because of its appropriate sol-gel transition temperature.

We measured and plotted the drug release profile (Figure 3) to observe the release of NEP1- 
244

245

246

247

248

249

250

251

252

253

254

255

256

257

258

259

260

261

262

263

264

265

266

267

268

269

270

on day 1 , and about $70 \%$ of NEP1-40 had been released within 14 days in a relatively smooth sustained-release trend. These findings indicate that the hydrogel can sustain drug release for at least 14 days.

\subsection{Treatment with the NEPI-40-loaded hydrogel sustained-release system significantly} improves the function of biceps brachii on the injured side after nerve root replantation for BPA

Steward O et al. showed that early application of NEP1-40 for 14 days can promote the recovery of spinal cord injury (Steward et al. 2008). And considering that the recovery period of brachial plexus injury (6-8 weeks) is shorter than that of spinal cord injury, and the time required for spinal cord axons to cross the spinal cord-peripheral nerve junction is shorter than that of spinal cord injury, therefore, we consider 14 days as the appropriate treatment time in our experiment (Nakamura et al. 2011a; Xu et al. 2017a; Zhai \& Feng 2019).

BPA model is confirmed by TGT as all the affected upper limb of animals found to receive grade of 0 on postoperative days 1 and 2 (Bertelli \& Mira 1993a). This suggested that we successfully established the BPA model. Recovery of nerve function was the most important goal of our study. First, we investigated whether the NEP1-40 hydrogel sustained-release treatment improved the recovery of motor function in rats. We found that the NEP1-40 hydrogel sustained-release system promoted motor function of the upper limbs after nerve root replantation for BPA. At 2-6 weeks after injury, TGT scores were higher in the NEP1-40-loaded hydrogel group than those in the control, blank hydrogel, and NEP1-40 groups. At 2-3 weeks after injury, TGT scores were significantly higher in the NEP1-40-loaded hydrogel group than those in the other groups, indicating a rapid recovery of nerve function. At 4 weeks after injury, TGT scores were rapidly increased in the four groups, and this increase was faster in the NEP140 and the NEP1-40-loaded hydrogel groups than in the other groups (Figure 4A). At 6 weeks post-surgery, the average TGT scores were $3.25,3.75,4.25$, and 4.63 in the control, blank hydrogel, NEP1-40, and NEP1-40-loaded hydrogel groups, respectively (Figures 4B and C). TGT scores were significantly higher in the NEP1-40 (Figure 4B; *P < 0.05) and NEP1-40- 
271 loaded hydrogel group (Figure 4B; **P $<0.01$ ) than in the control group. TGT scores in the

272 blank hydrogel group were also higher than those in the control group, but this difference was

273 not obvious. Therefore, treatment with the NEP1-40-loaded hydrogel sustained-release system

274 significantly increased the TGT scores of the affected limbs, indicating that nerve function 275 recovered well (GrandPré et al. 2002).

276 3.3 Treatment with the NEPI-40-loaded hydrogel sustained-release system significantly 277 increases the survival rate of anterior horn motoneurons and the number of functional

\section{motoneurons on the injured side}

Neuronal survival is the basis of nerve regeneration. In this study, we observed the number of anterior horn motoneurons and functional motoneurons in the injured spinal cord. The spinal anterior horn motoneurons labeled by ChAT, a specific antibody which can help to distinguish between neuron and other tissues, were significantly bigger (their morphology was similar to normal) in the NEP1-40-loaded hydrogel group than in the other groups at 6 weeks post-surgery. Therefore, neuronal function was better preserved in the NEP1-40-loaded hydrogel group than in the remaining groups. The cell number ratios between the injured and healthy sides were 51.33\%, $57.33 \%, 72.33 \%$, and 75.67\% (Figure 5E; $* \mathrm{P}<0.05$ ). Ratios were higher in the NEP1-40 and NEP1-40-loaded hydrogel groups than in the control group (Figure 5E; *P $<0.05$ ).

FG nerve retrograde labeling of the $\mathrm{C} 5-\mathrm{C} 8$ segments revealed that the number of motoneurons in the spinal cord and their size increased significantly after treatment with the NEP1-40-loaded hydrogel sustained-release system (Figures 5F-J; * $\mathrm{P}<0.05$, ** $\mathrm{P}<0.01$ ), indicating that the number of neurons involved in motor function was increased. Due to the fact that the anterior horn motor neurons are limited to the anterior horn of the spinal cord, so the range is small. There are no positive cells in other places, which are blank. So, in order to display the cells more intuitively, parts with obvious cells are only put there (the original figures could be seen in the supplementary file). Furthermore, the number of functional motoneurons was higher in the NEP1-40 treatment group than in the control treatment group (Figure 5J; * $\mathrm{P}<$ 0.05). The number of functional motoneurons was slightly but insignificantly increased in the 
blank hydrogel group as compared to the control group.

Regenerated axons need to pass through or bypass scar tissue in the injured area. Scar tissues contain various nerve growth inhibitory factors that make axonal regeneration tortuous and increase the probability of neural mismatch. Thus, the efficiency of axonal regeneration is decreased, and the difficulty of nerve regeneration is increased (Cullheim et al. 1999; Soderblom et al. 2013). After BPA, nerve root replantation initiates axon regeneration and increases the survival rate of numerous neurons (Gu et al. 2004). Furthermore, axons grow rapidly, and the survival rate of neurons is greatly improved ( $\mathrm{Li}$ et al. 2015). Our results revealed that NEPI-40 promoted axonal growth, thereby preventing necrosis of neurons. Therefore, NEP1-40 had a certain neuroprotective effect, providing a good foundation for nerve regeneration. In addition, we assessed the number of functional motoneurons: the number of FG-labeled neurons was significantly higher after treatment with the NEP1-40-loaded hydrogel sustained-release system than after control treatment (the control group) (Figure 5J; ** $\mathrm{P}<0.01$ ) or the NEP1-40-alone treatment (the NEP1-40 group) (Figure 5J, * P $<0.05$ ). This finding indicates that hydrogel and NEPl-40 treatment have synergistic effects, and more neurons participate in functional recovery, thereby promoting the restoration of nerve function.

\subsection{Treatment with the NEPI-40-loaded hydrogel sustained-release system can promote the} regeneration of bicep muscle fibers and MEPs on the injured side

After BPA, denervation of muscle tissues leads to atrophy and dysfunction (Duijnisveld et al. 2017). Regeneration and growth of axons into target muscles allows for the recovery of atrophic muscles, fibrosis reversion of muscle fibers, and thickening of the diameter of muscle fibers to adapt to functional changes. MEPs in the muscles are concurrently re-innervated, and the MEP size is enlarged, recovering the original structure (Ali et al. 2016).

MEPs can be an important indicator of muscle function recovery after reinnervation (Wang et al. 2018). Measurements of the area of motor endplates in the injured biceps brachii at 6 weeks post-surgery revealed that MEP area increased more significantly after treatment with the NEP140-loaded hydrogel sustained-release system than with the other treatments (other groups). 
325

326

327

328

329

330

331

332

333

334

335

336

337

338

339

340

341

342

343

344

345

346

347

348

349

350

351

Additionally, MEP structure was clearer and more complex after treatment with the NEP1-40loaded hydrogel sustained-release system than after other treatments. The area of MEPs in the NEP1-40-loaded hydrogel group was $27.3 \%$ higher than in the control group (Figure 6A-D, I; ** $\mathrm{P}<0.01$ ), indicating that the NEP1-40-loaded hydrogel sustained-release system provides an anatomical basis for functional recovery. The size and complexity of MEPs were higher, and the area of MEPs was $18.7 \%$ higher in the NEP1-40 treatment group than in the control group (Figures 6A-D, I; *P $<0.05$ ), indicative of the effectiveness of NEP1-40 treatment.

Regenerated axons enter MEPs to trigger an increase in the volume of target muscle fibers, thereby leading to functional recovery. We performed hematoxylin-eosin staining of the muscle fibers of the injured biceps brachii at 6 weeks post-surgery and assessed fiber diameter. We found that muscle fiber diameter was the highest in the NEP1-40-loaded hydrogel group. Additionally, the muscle fiber diameter was $28.8 \%$ higher in the NEP1-40-loaded hydrogel group and 16\% higher in the NEP1-40 group than in the control group (Figures 6E-H, J; ****P < $0.0001, * * \mathrm{P}<0.01$, respectively). The increase in muscle fiber diameter indicates a good recovery of function. However, muscle fiber diameter in the blank hydrogel group was only slightly higher than in the control group.

\section{Conclusion}

Our findings indicate that the sustained release of NEP1-40 from the PLGA-PEG-PLGA hydrogel promotes nerve regeneration and recovery of motor function in BPA rats, promotes axonal growth, and protects neurons. The PLGA-PEG-PLGA hydrogel acts as a slow-release carrier and concurrently fills defects, thereby providing favorable conditions for nerve regeneration. Therefore, the combination of NEP1-40 and PLGA-PEG-PLGA hydrogel can promote axonal growth and recovery of nerve function. The present findings shed light on the potential application of the PLGA-PEG-PLGA hydrogel for the treatment of brachial plexus root avulsion. 


\section{Funding}

353 This study was supported by the Jilin Provincial Educational 135 Science and Technology

354 Project, JJKH20190047KJ.

\section{Data Availability}

356 The following information was supplied regarding data availability: All data for the different 357 figures are available in the Supplemental Files, named date-58944 ZIP.

\section{Ethical statement}

359 This study was approved by the Research Ethics Committee of the Second Hospital of Jilin 360 University.

\section{Disclosure of interest}

362 There are no conflicts of interest to declare.

\section{Authors' contributions}

364 All authors substantially contributed to the manuscript and approved the final manuscript. 365 Tianwen Sun and Zhe Zhu contributed equally to this work, and share co-corresponding 366 authorship.

\section{References}

Ali ZS, Johnson VE, Stewart W, Zager EL, Xiao R, Heuer GG, Weber MT, Mallela AN, and Smith DH. 2016. Neuropathological characteristics of brachial plexus avulsion injury with and without concomitant spinal cord injury. Journal of Neuropathology \& Experimental Neurology 75:69-85.

Baazil H, Dalemans A, De Smet L, and Degreef I. 2015. Comparison of surgical treatments for mucous cysts of the distal interphalangeal joint. Acta Orthop Belg 81:213-217.

Bertelli JA, and Mira J-C. 1993a. Behavioral evaluating methods in the objective clinical assessment of motor function after experimental brachial plexus reconstruction in the rat. Journal of neuroscience methods 46:203-208.

Bertelli JA, and Mira JC. 1993b. Behavioral evaluating methods in the objective clinical assessment of motor function after experimental brachial plexus reconstruction in the rat. $J$ Neurosci Methods 46:203-208. 10.1016/0165-0270(93)90068-3

Birrell MT, and Fuller A. 2019. Twice daily cefazolin is effective for treatment of serious methicillin-sensitive Staphylococcus aureus infection in an outpatient parenteral antimicrobial therapy program. Ther Adv Infect Dis 6:2049936119882847. 10.1177/2049936119882847

Cao Y, Shumsky J, Sabol M, Kushner R, Strittmatter S, Hamers F, Lee D, Rabacchi S, and Murray M. 2008. Nogo66 receptor antagonist peptide (NEP1-40) administration promotes functional recovery and axonal growth 
after lateral funiculus injury in the adult rat. Neurorehabilitation and Neural Repair 22:262-278.

Carlstedt T. 2008. Root repair review: basic science background and clinical outcome. Restorative neurology and neuroscience 26:225-241.

Chen MS, Huber AB, van der Haar ME, Frank M, Schnell L, Spillmann AA, Christ F, and Schwab ME. 2000. Nogo-A is a myelin-associated neurite outgrowth inhibitor and an antigen for monoclonal antibody IN-1. Nature 403:434-439.

Ci T, Chen L, Yu L, and Ding J. 2014. Tumor regression achieved by encapsulating a moderately soluble drug into a polymeric thermogel. Sci Rep 4:5473. 10.1038/srep05473

Ciaramitaro P, Padua L, Devigili G, Rota E, Tamburin S, Eleopra R, Cruccu G, Truini A, and Society NpsigotIN. 2017. Prevalence of neuropathic pain in patients with traumatic brachial plexus injury: A multicenter prospective hospital-based study. Pain Medicine 18:2428-2432.

Cullheim S, Carlstedt T, and Risling M. 1999. Axon regeneration of spinal motoneurons following a lesion at the cord-ventral root interface. Spinal cord 37:811-819.

Ding L, Zhu Z, Wang Y, Zeng L, Wang T, Luo J, Zou T-B, Li R, Sun X, Zhou G, Liu X, and Wu H-F. 2019. LINGO-1 shRNA Loaded by Pluronic F-127 Promotes Functional Recovery After Ventral Root Avulsion. Tissue Engineering Part A 25:1381-1395. 10.1089/ten.tea.2018.0282

Duijnisveld BJ, Henseler JF, Reijnierse M, Fiocco M, Kan HE, and Nelissen RG. 2017. Quantitative Dixon MRI sequences to relate muscle atrophy and fatty degeneration with range of motion and muscle force in brachial plexus injury. Magnetic resonance imaging 36:98-104.

Eggers R, Tannemaat M, Ehlert E, and Verhaagen J. 2010. A spatio-temporal analysis of motoneuron survival, axonal regeneration and neurotrophic factor expression after lumbar ventral root avulsion and implantation. Experimental neurology 223:207-220.

Faglioni W, Siqueira MG, Martins RS, Heise CO, and Foroni L. 2014. The epidemiology of adult traumatic brachial plexus lesions in a large metropolis. Acta neurochirurgica 156:1025-1028.

Gibon E, Romana C, Vialle R, and Fitoussi F. 2016. Isolated C5-C6 avulsion in obstetric brachial plexus palsy treated by ipsilateral $\mathrm{C} 7$ neurotization to the upper trunk: outcomes at a mean follow-up of 9 years. Journal of Hand Surgery (European Volume) 41:185-190.

GrandPré T, Li S, and Strittmatter SM. 2002. Nogo-66 receptor antagonist peptide promotes axonal regeneration. Nature 417:547-551.

Gu HY, Chai H, Zhang JY, Yao ZB, Zhou LH, Wong WM, Bruce I, and Wu WT. 2004. Survival, regeneration and functional recovery of motoneurons in adult rats by reimplantation of ventral root following spinal root avulsion. European Journal of Neuroscience 19:2123-2131.

Huang C, Fu C, Qi ZP, Guo WL, You D, Li R, and Zhu Z. 2020. Localised delivery of quercetin by thermo-sensitive PLGA-PEG-PLGA hydrogels for the treatment of brachial plexus avulsion. Artif Cells Nanomed Biotechnol 48:1010-1021. 10.1080/21691401.2020.1770265

Kempf A, and Schwab ME. 2013. Nogo-A represses anatomical and synaptic plasticity in the central nervous system. Physiology 28:151-163.

Kwok-FaiSo, and Xiao-MingXu. 2015. Neural regeneration: Neural regeneration.

Lang E, Schlegel N, Sendtner M, and Asan E. 2005. Effects of root replantation and neurotrophic factor treatment on long-term motoneuron survival and axonal regeneration after $\mathrm{C} 7$ spinal root avulsion. Experimental neurology 194:341-354. 
426

427

428

429

430

431

432

433

434

435

436

437

438

439

440

441

442

443

444

445

446

447

448

449

450

451

452

453

454

455

456

457

458

459

460

461

462

463

464

465

466

Li H, Wong C, Li W, Ruven C, He L, Wu X, Lang BT, Silver J, and Wu W. 2015. Enhanced regeneration and functional recovery after spinal root avulsion by manipulation of the proteoglycan receptor PTP $\sigma$. Scientific reports 5:14923.

Li S, and Strittmatter SM. 2003. Delayed systemic Nogo-66 receptor antagonist promotes recovery from spinal cord injury. Journal of Neuroscience 23:4219-4227.

Limthongthang R, Bachoura A, Songcharoen P, and Osterman AL. 2013. Adult brachial plexus injury: evaluation and management. Orthopedic Clinics 44:591-603.

Luo J, Borgens R, and Shi R. 2004. Polyethylene glycol improves function and reduces oxidative stress in synaptosomal preparations following spinal cord injury. Journal of neurotrauma 21:994-1007.

Macaya D, and Spector M. 2012. Injectable hydrogel materials for spinal cord regeneration: a review. Biomedical materials 7:012001.

Meng C, Yang X, Liu Y, Zhou Y, Rui J, Li S, Xu C, Zhuang Y, Lao J, and Zhao X. 2019. Decreased expression of lncRNA Malat1 in rat spinal cord contributes to neuropathic pain by increasing neuron excitability after brachial plexus avulsion. J Pain Res 12:1297-1310. 10.2147/JPR.S195117

Meng XL, Fu P, Wang L, Yang X, Hong G, Zhao X, and Lao J. 2020. Increased EZH2 Levels in Anterior Cingulate Cortex Microglia Aggravate Neuropathic Pain by Inhibiting Autophagy Following Brachial Plexus Avulsion in Rats. Neurosci Bull 36:793-805. 10.1007/s12264-020-00502-w

Mingorance A, Solé M, Munetón V, Martínez A, Nieto-Sampedro M, Soriano E, and del Río JA. 2006. Regeneration of lesioned entorhino-hippocampal axons in vitro by combined degradation of inhibitory proteoglycans and blockade of Nogo-66/NgR signaling. The FASEB journal 20:491-493.

Nakamura Y, Fujita Y, Ueno M, Takai T, and Yamashita T. 2011a. Paired immunoglobulin-like receptor B knockout does not enhance axonal regeneration or locomotor recovery after spinal cord injury. $J$ Biol Chem 286:1876-1883. 10.1074/jbc.M110.163493

Nakamura Y, Fujita Y, Ueno M, Takai T, and Yamashita T. 2011b. Paired immunoglobulin-like receptor B knockout does not enhance axonal regeneration or locomotor recovery after spinal cord injury. Journal of Biological Chemistry 286:1876-1883.

National C, and Promising I. 2009. The National Academies Collection: Reports funded by National Institutes of Health - NCBI Bookshelf. National Academies Press.

Rong X, Ji Y, Zhu X, Yang J, Qian D, Mo X, and Lu Y. 2019. Neuroprotective effect of insulin-loaded chitosan nanoparticles/PLGA-PEG-PLGA hydrogel on diabetic retinopathy in rats. International journal of nanomedicine 14:45.

Shin AY, Spinner RJ, Steinmann SP, and Bishop AT. 2005. Adult traumatic brachial plexus injuries. JAAOSJournal of the American Academy of Orthopaedic Surgeons 13:382-396.

Shrestha B, Coykendall K, Li Y, Moon A, Priyadarshani P, and Yao L. 2014. Repair of injured spinal cord using biomaterial scaffolds and stem cells. Stem cell research \& therapy 5:91.

Soderblom C, Luo X, Blumenthal E, Bray E, Lyapichev K, Ramos J, Krishnan V, Lai-Hsu C, Park KK, and Tsoulfas P. 2013. Perivascular fibroblasts form the fibrotic scar after contusive spinal cord injury. Journal of Neuroscience 33:13882-13887.

Steward O, Sharp K, Yee KM, and Hofstadter M. 2008. A re-assessment of the effects of a Nogo-66 receptor antagonist on regenerative growth of axons and locomotor recovery after spinal cord injury in mice. Exp Neurol 209:446-468. 10.1016/j.expneurol.2007.12.010

Peer] reviewing PDF | (2021:03:58944:2:1:NEW 14 Sep 2021) 
Teixeira MJ, da Paz MGdS, Bina MT, Santos SN, Raicher I, Galhardoni R, Fernandes DT, Yeng LT, Baptista AF, and De Andrade DC. 2015. Neuropathic pain after brachial plexus avulsion-central and peripheral mechanisms. BMC neurology 15:73.

Tetsuro, and Kusaba. 2009. Safety and Efficacy of Cefazolin Sodium in the Management of Bacterial Infection and in Surgical Prophylaxis. Clinical Medicine Therapeutics 1.

Wang F, Liang Z, Hou Q, Xing S, Ling L, He M, Pei Z, and Zeng J. 2007. Nogo-A is involved in secondary axonal degeneration of thalamus in hypertensive rats with focal cortical infarction. Neuroscience letters 417:255260.

Wang Z, Fan J, Yang X, Zhang W, Zhang P, and Jiang B. 2018. The neural regeneration effect of chitin biological absorbable tubes bridging sciatic nerve defects with sural nerve grafts. American journal of translational research 10:2362.

Xu J, He J, He H, Peng R, and Xi J. 2017a. Comparison of RNAi NgR and NEP1-40 in Acting on Axonal Regeneration After Spinal Cord Injury in Rat Models. Mol Neurobiol 54:8321-8331. 10.1007/s12035-0160315-3

Xu J, He J, He H, Peng R, and Xi J. 2017b. Comparison of RNAi NgR and NEP1-40 in Acting on Axonal Regeneration After Spinal Cord Injury in Rat Models. Molecular neurobiology 54:8321-8331.

Young W. 1996. Spinal cord regeneration. 273:451-451.

Zhai ZY, and Feng J. 2019. Constraint-induced movement therapy enhances angiogenesis and neurogenesis after cerebral ischemia/reperfusion. Neural Regen Res 14:1743-1754. 10.4103/1673-5374.257528

Zhang K, Tang X, Zhang J, Lu W, Lin X, Zhang Y, Tian B, Yang H, and He H. 2014a. PEG-PLGA copolymers: Their structure and structure-influenced drug delivery applications. Journal of Controlled Release 183:7786. 10.1016/j.jconrel.2014.03.026

Zhang K, Tang X, Zhang J, Lu W, Lin X, Zhang Y, Tian B, Yang H, and He H. 2014b. PEG-PLGA copolymers: Their structure and structure-influenced drug delivery applications. Journal of Controlled release 183:7786.

Zhang Z, Ni J, Chen L, Yu L, Xu J, and Ding J. 2011. Biodegradable and thermoreversible PCLA-PEG-PCLA hydrogel as a barrier for prevention of post-operative adhesion. Biomaterials 32:4725-4736.

Zhao J, Guo B, and Ma PX. 2014. Injectable alginate microsphere/PLGA-PEG-PLGA composite hydrogels for sustained drug release. Rsc Advances 4:17736-17742.

Zhou Y, Liu P, Rui J, Zhao X, and Lao J. 2017. The Associated Factors and Clinical Features of Neuropathic Pain After Brachial Plexus Injuries. The Clinical journal of pain 33:1030-1036.

\section{Tables}

Table 1. Grouping and treatments.

\section{Figure lengths}

Figure 1. The ${ }^{1} \mathrm{H}$ NMR spectrum of the PLGA-PEG-PLGA copolymer in $\mathrm{CDCl}_{3}$. The characteristic signals appearing at 5.2, 4.8, 3.5, and $1.5 \mathrm{ppm}$ represent the $\mathrm{CH}$ of $\mathrm{LA}, \mathrm{CH}_{2}$ of GA, $\mathrm{CH}_{2}$ of $\mathrm{PEG}$, and $\mathrm{CH}_{3}$ of $\mathrm{LA}$, respectively. 
506 Figure 2. Sol-to-gel transition temperature measurement of PLGA-PEG-PLGA copolymers

507

508

509

510

511

512

513

514

515

516

517

518

519

520

521

522

523

524

525

526

527

528

529

530

531

532 determined using the vial inversion test and phase diagram of the sol-gel-precipitation transition as a function of PLGA-PEG-PLGA concentration. A. Copolymers in the sol state; B. Copolymers in the gel state; C: Copolymers in the precipitate state. PLGA-PEG-PLGA: poly (D, L-lactide-co-glycolide)-poly (ethylene glycol)-poly (D, L-lactide-co-glycolide).

Figure 3. Cumulative release profile of NEP1-40. On day 1, about 37\% NEP1-40 was released from the PLGA-PEG-PLGA hydrogel. During days 2-14, 70\% of the NEP1-40 was steadily released. Data are presented as means \pm standard errors $(n=3)$.

Figure 4. TGT scores $(n=8)$ of the control, blank hydrogel, NEP1-40, and NEP1-40-loaded hydrogel groups at 2-6 weeks post-surgery. (A) TGT scores in each group at 2-6 weeks postsurgery. (B) TGT scores in each group at 6 weeks post-surgery $(* \mathrm{P}<0.05$, ** $\mathrm{P}<0.01)$. (C) Representative images of the rats at 6 weeks post-surgery. The rat in the control group did not touch its eyes with the affected upper limb (right), but the rat in the Gel+NEP1-40 group reached the ears with the affected upper limb (right). Arrow red: right upper limb; arrow blue: ear.

Figure 5. Survival rates of anterior horn motoneurons and the number of FG-labeled motoneurons on the injured side C6 segment at 6 weeks post-surgery. (A-D) ChAT (+) neurons (red) of the C6 spinal segment in the control (A), blank hydrogel (B), NEP1-40 (C), and NEP140-loaded hydrogel (D) groups, respectively (scale bar $=100 \mu \mathrm{m})$. (E) Survival rates of anterior horn motoneurons of the $\mathrm{C} 6$ segment $(\mathrm{n}=3, * \mathrm{P}<0.05)$. (F-I) FG nerve retrograde labeling of anterior horn motoneurons of the C5-C8 segments in the control (F), blank hydrogel (G), NEP1$40(\mathrm{H})$, and NEPl-40-loaded hydrogel (I) groups, respectively $(\mathrm{n}=3$, scale bar $=100 \mu \mathrm{m})$. (J) The number of FG-labeled anterior horn motoneurons of the $\mathrm{C} 5-\mathrm{C} 8$ segments $(\mathrm{n}=3, * \mathrm{P}<0.05$, ** $\mathrm{P}<0.01)$.

Figure 6. Immunofluorescence staining of MEPs of the injured biceps brachii and hematoxylineosin staining of muscle fibers in the control (A), blank hydrogel (B), NEP1-40 (C), and NEP140-loaded hydrogel groups (D) at 6 weeks post-surgery. A-D: MEPs of the injured biceps brachii in each group (red) $(\mathrm{n}=4$, scale bar $=20 \mu \mathrm{m})$. E-H: Hematoxylin-eosin staining of the biceps 
533 brachii in in the control (E), blank hydrogel (F), NEP1-40 (G), and NEP1-40-loaded hydrogel (H) 534 groups, respectively $(\mathrm{n}=4$, scale bar $=20 \mu \mathrm{m})$. I: The area of MEPs of the biceps brachii on the 535 injured side at 6 weeks post-surgery $(* \mathrm{P}<0.05, * * \mathrm{P}<0.01)$. J: Muscle fiber diameter of the 536 biceps brachii on the injured side at 6 weeks post-surgery $(* * \mathrm{P}<0.01, * * * * \mathrm{P}<0.0001)$. 
Figure 1

The ${ }^{1} \mathrm{H}$ NMR spectrum of the PLGA-PEG-PLGA copolymer in $\mathrm{CDCl}_{3}$.

Figure 1. The ${ }^{1} \mathrm{H}$ NMR spectrum of the PLGA-PEG-PLGA copolymer in $\mathrm{CDCl}_{3}$. The characteristic signals appearing at $5.2,4.8,3.5$, and $1.5 \mathrm{ppm}$ represent the $\mathrm{CH}$ of $\mathrm{LA}, \mathrm{CH}_{2}$ of $\mathrm{GA}, \mathrm{CH}_{2}$ of $\mathrm{PEG}$, and $\mathrm{CH}_{3}$ of $\mathrm{LA}$, respectively.

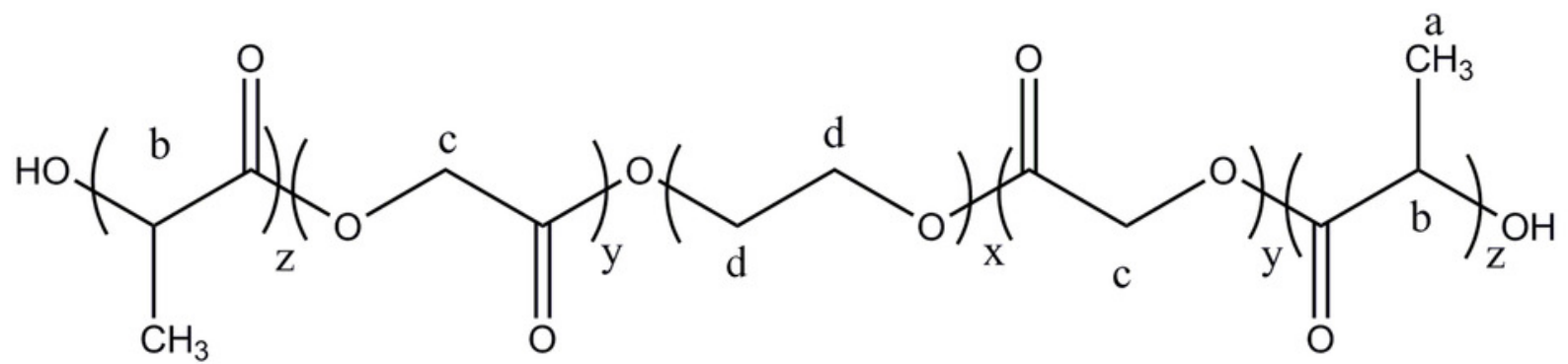

a

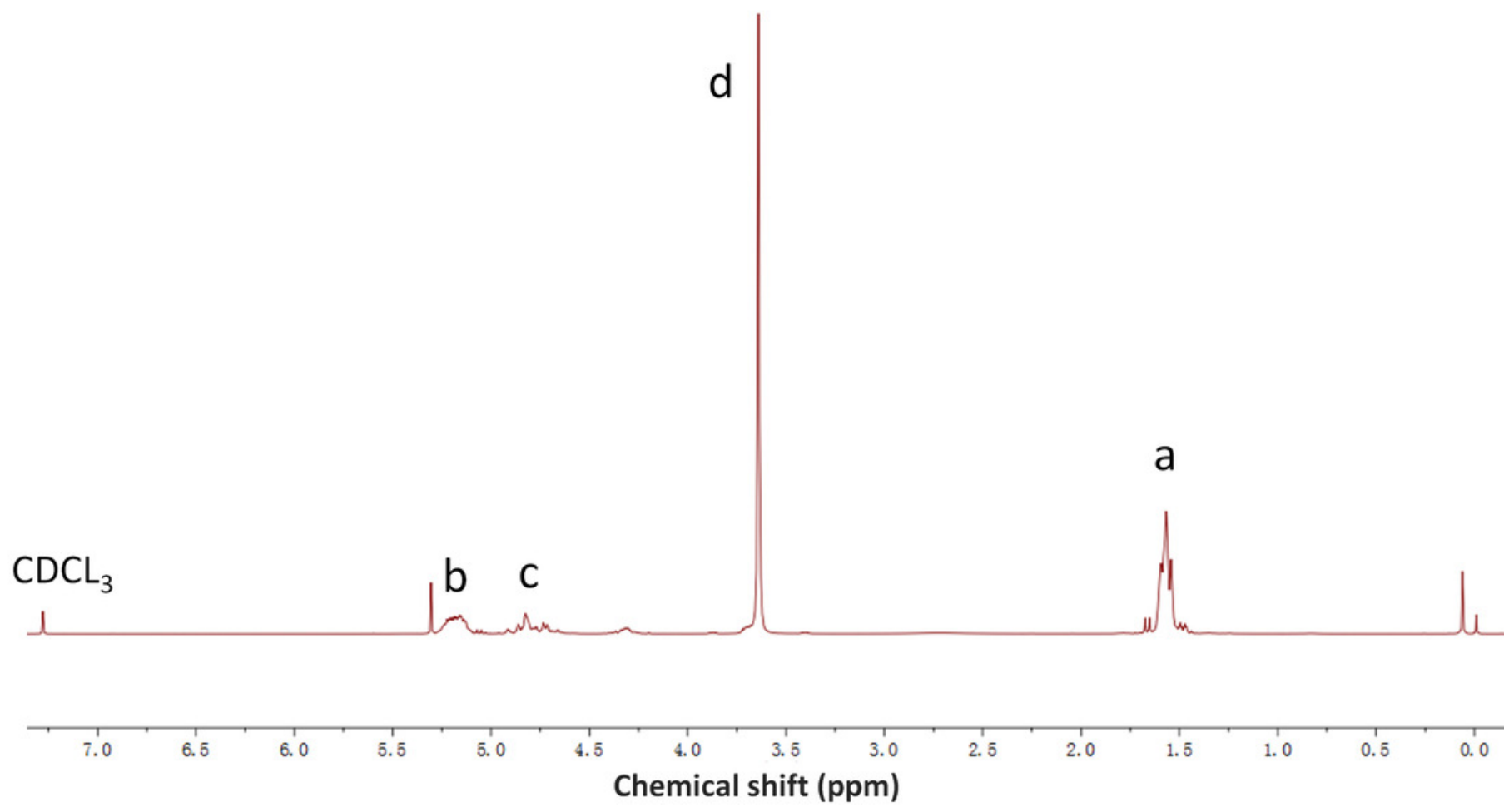




\section{Figure 2}

Sol-to-gel transition temperature measurement of PLGA-PEG-PLGA copolymers determined using the vial inversion test and phase diagram of the sol-gel-precipitation transition as a function of PLGA-PEG-PLGA concentration.

Figure 2. Sol-to-gel transition temperature measurement of PLGA-PEG-PLGA copolymers determined using the vial inversion test and phase diagram of the sol-gel-precipitation transition as a function of PLGA-PEG-PLGA concentration. A. Copolymers in the sol state; B. Copolymers in the gel state; C: Copolymers in the precipitate state. PLGA-PEG-PLGA: poly (D, L-lactide-co-glycolide)-poly (ethylene glycol)-poly (D,L-lactide-co-glycolide). 


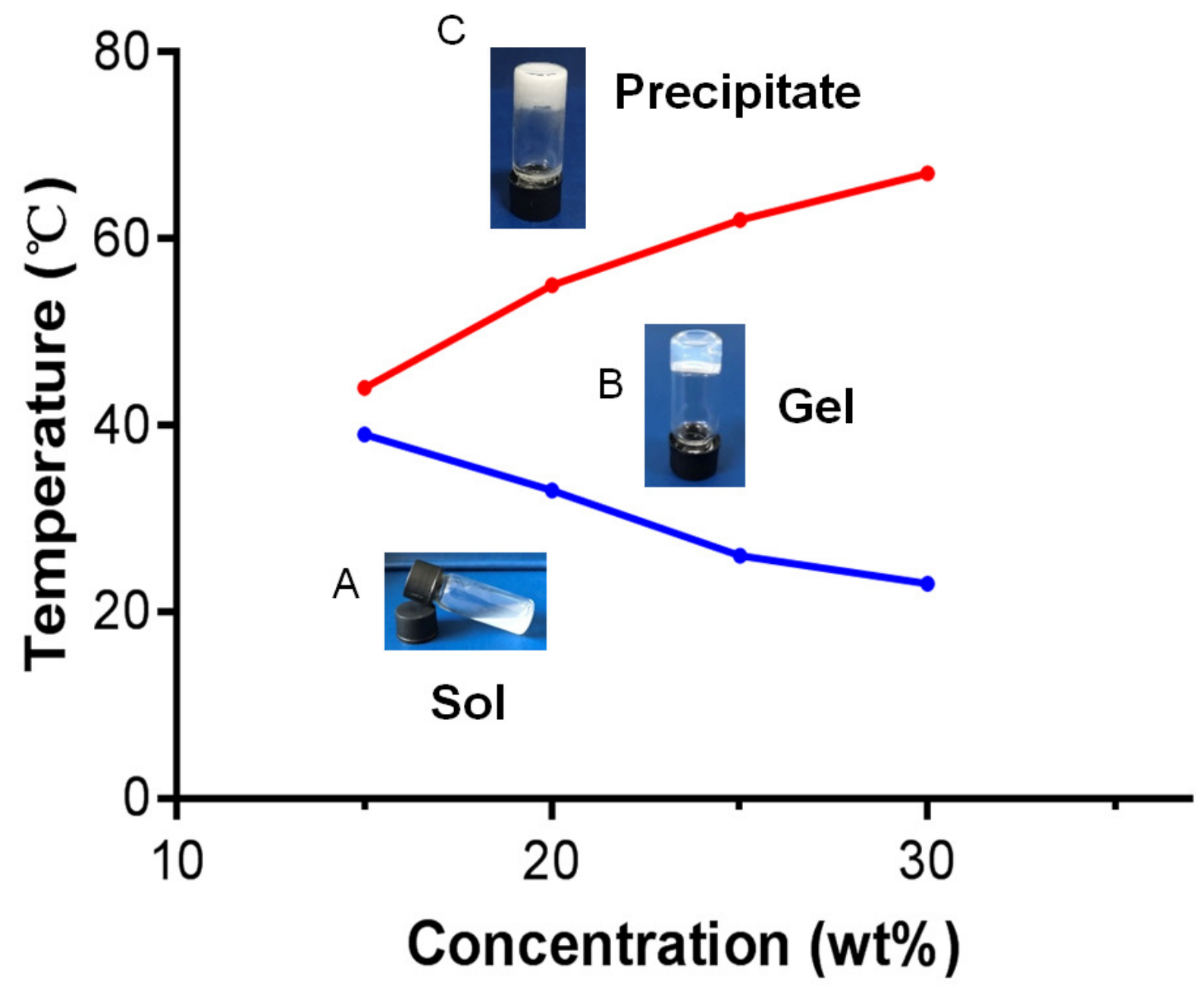


Figure 3

Cumulative release profile of NEP1-40.

Figure 3. Cumulative release profile of NEP1-40. On day 1 , about 37\% NEP1-40 was released from the PLGA-PEG-PLGA hydrogel. During days $2-14,70 \%$ of the NEP1-40 was steadily released. Data are presented as means \pm standard errors $(n=3)$.

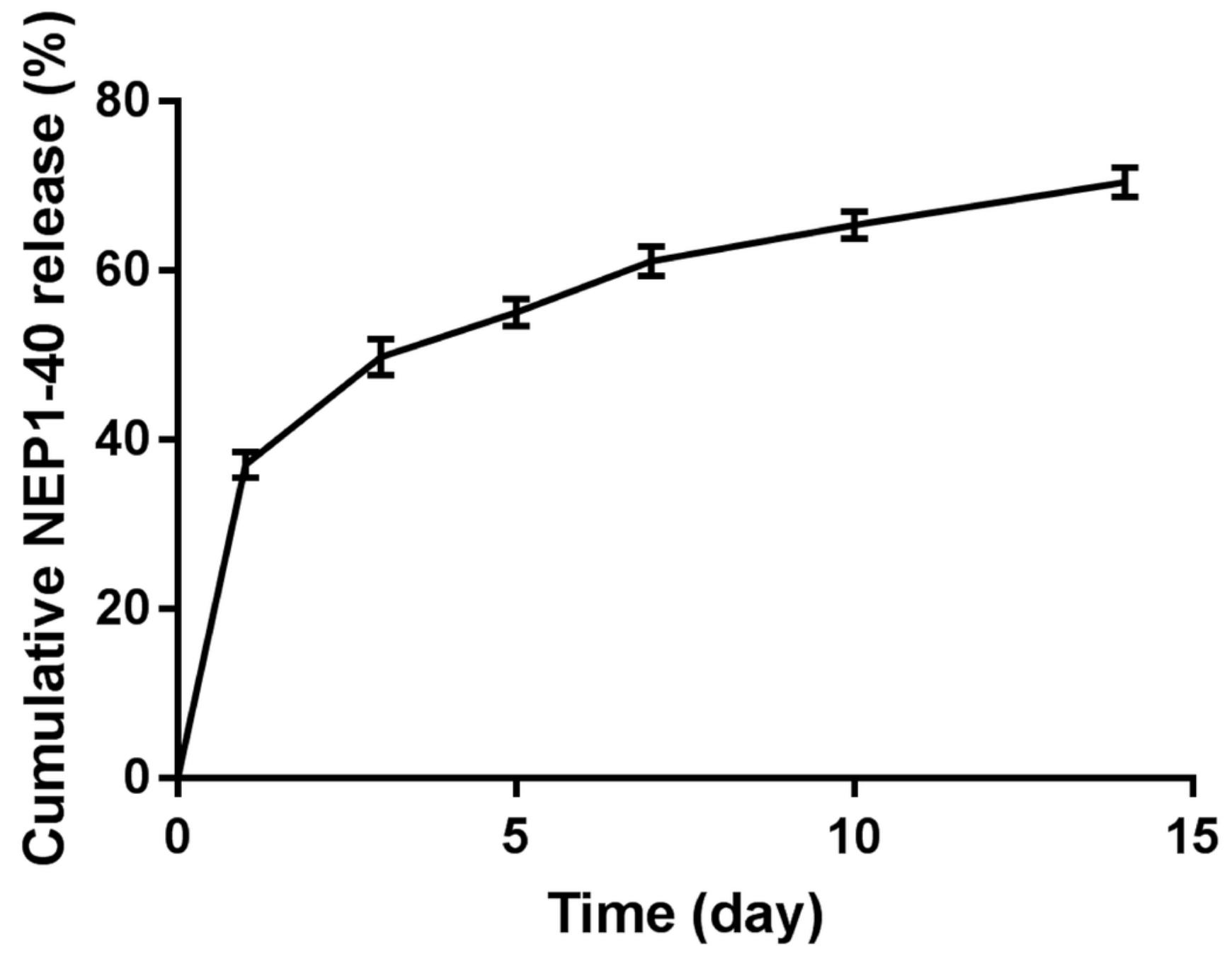




\section{Figure 4}

TGT scores $(n=8)$ of the control, blank hydrogel, NEP1-40, and NEPI-40-loaded hydrogel groups at 2-6 weeks post-surgery.

Figure 4. TGT scores $(n=8)$ of the control, blank hydrogel, NEP1-40, and NEPI-40-loaded hydrogel groups at 2-6 weeks post-surgery. (A) TGT scores in each group at 2-6 weeks postsurgery. (B) TGT scores in each group at 6 weeks post-surgery ( $* P<0.05, * * P<0.01$ ). (C) Representative images of the rats at 6 weeks post-surgery. The rat in the control group did not touch its eyes with the affected upper limb (right), but the rat in the Gel+NEP1-40 group reached the ears with the affected upper limb (right). Arrow red: right upper limb; arrow blue: ear. 
A

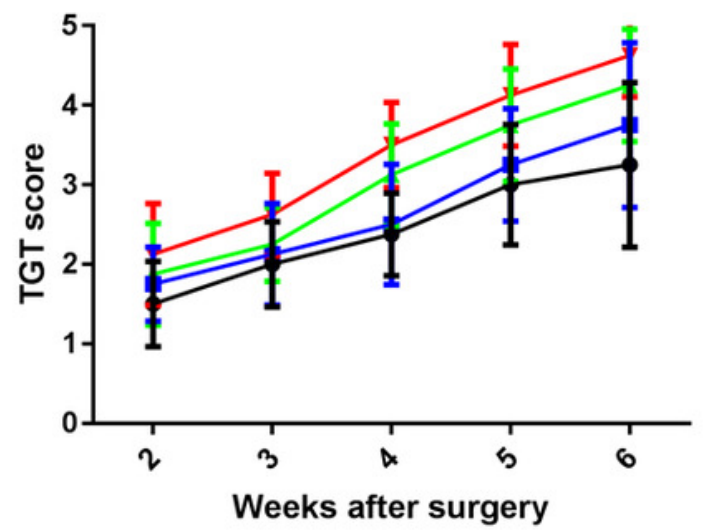

B

$\rightarrow$ Gel+NEP1-40

$₫$ NEP1-40

$\rightarrow$ Gel

$\rightarrow$ Control

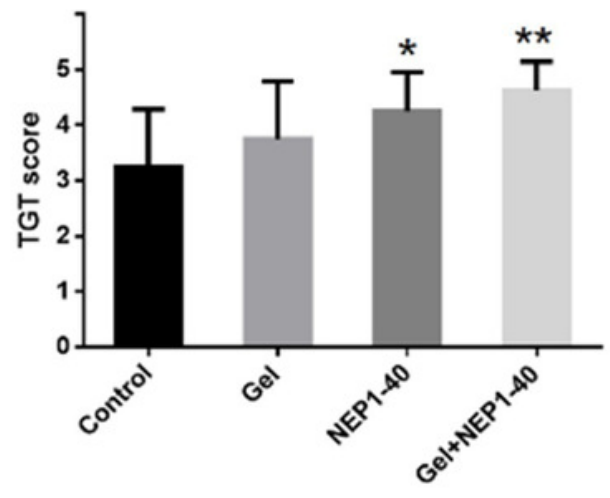

6 weeks after surgery

C

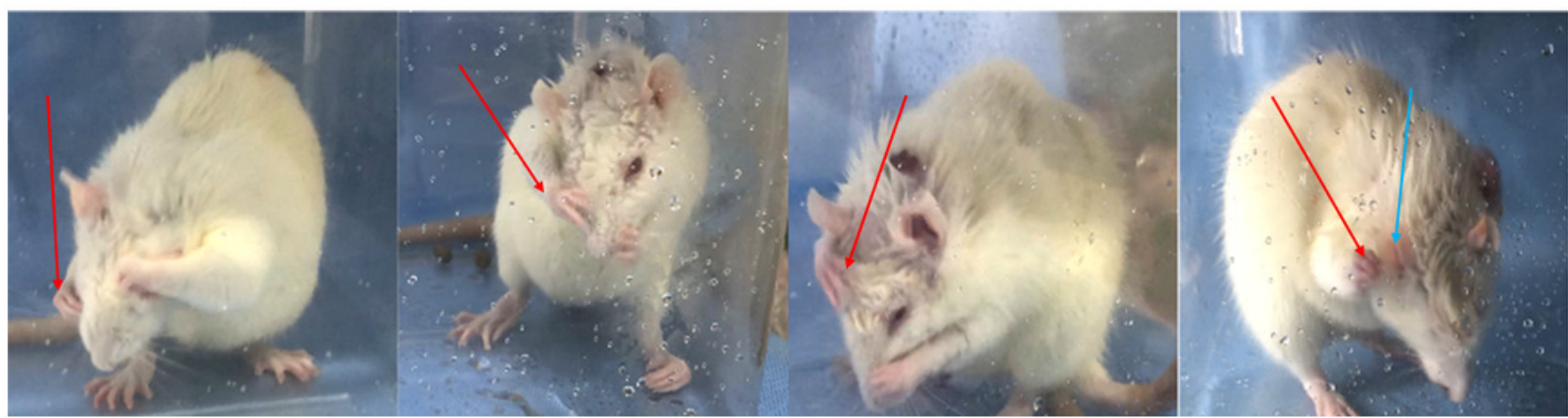

Control

Gel

NEP1-40

Gel+NEP1-40 


\section{Figure 5}

Survival rates of anterior horn motoneurons and the number of FG-labeled motoneurons on the injured side $\mathrm{C} 6$ segment at 6 weeks post-surgery.

Figure 5. Survival rates of anterior horn motoneurons and the number of FG-labeled motoneurons on the injured side $\mathrm{C} 6$ segment at 6 weeks post-surgery. (A-D) ChAT (+) neurons (red) of the C6 spinal segment in the control, blank hydrogel, NEP1-40, and NEPI-40loaded hydrogel groups, respectively (scale bar $=100 \mu \mathrm{m}$ ). (E) Survival rates of anterior horn motoneurons of the $\mathrm{C} 6$ segment $(\mathrm{n}=3, * \mathrm{P}<0.05)$. (F-I) FG nerve retrograde labeling of anterior horn motoneurons of the C5-C8 segments, respectively $(n=3$, scale bar $=100 \mu \mathrm{m})$. (J) The number of FG-labeled anterior horn motoneurons of the C5-C8 segments ( $\mathrm{n}=3, * \mathrm{P}<$ $0.05, * * \mathrm{P}<0.01)$. 

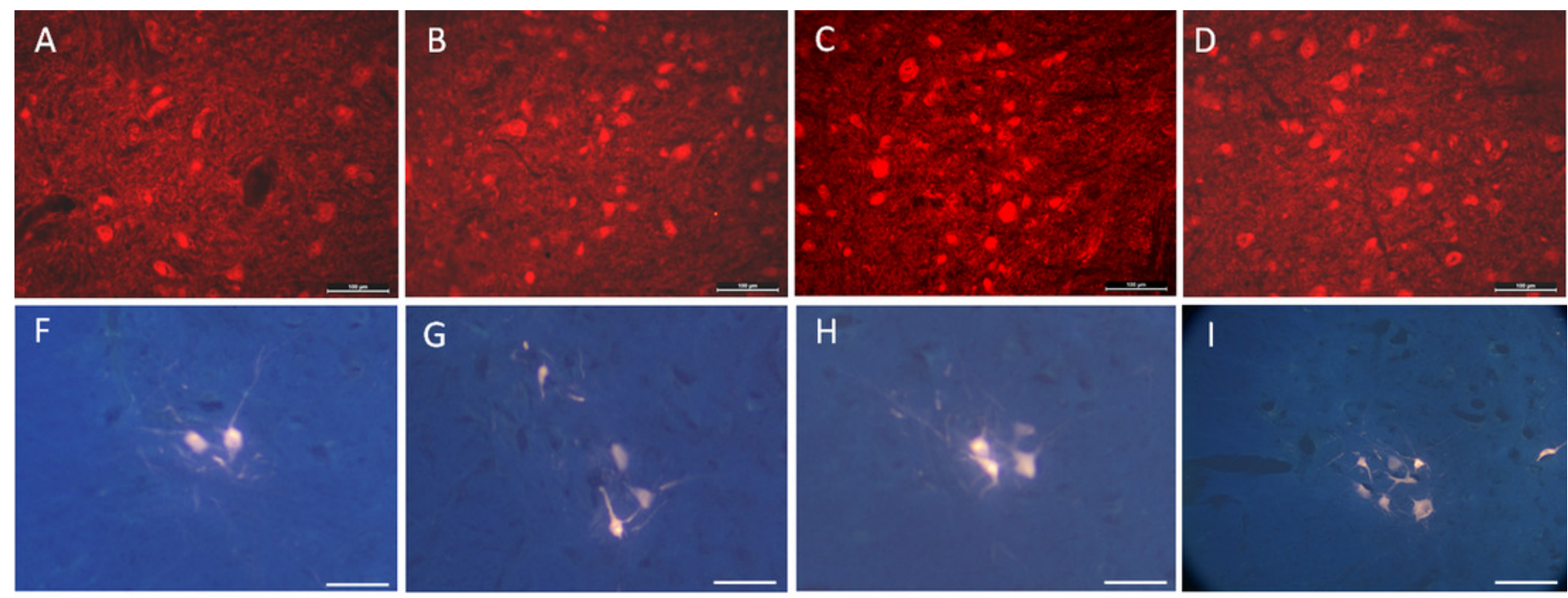

\section{E}

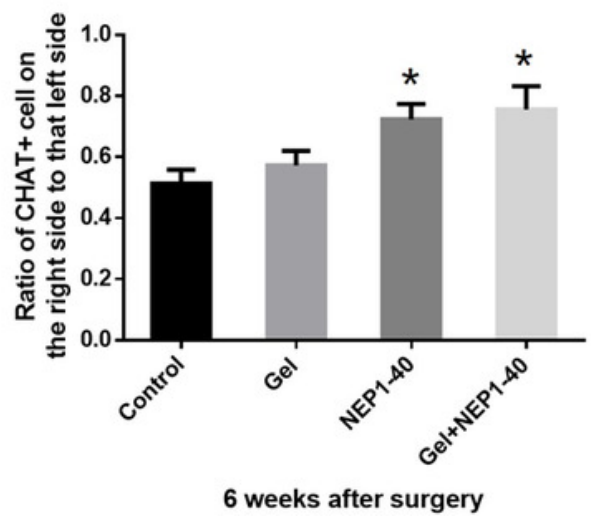

J

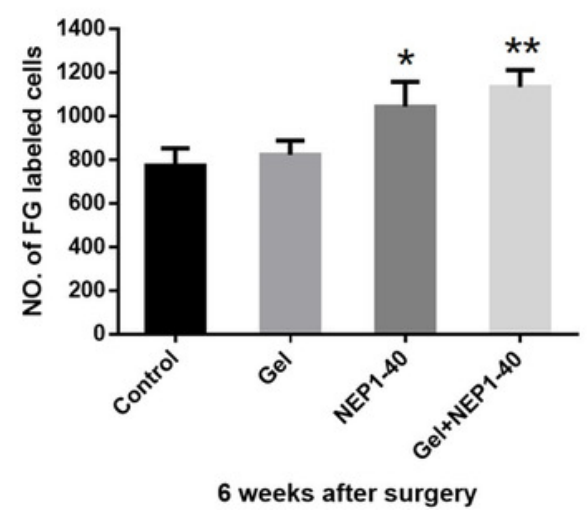




\section{Figure 6}

Immunofluorescence staining of MEPs of the injured biceps brachii and hematoxylineosin staining of muscle fibers in the control, blank hydrogel, NEP1-40, and NEPI-40loaded hydrogel groups at 6 weeks post-surgery.

Figure 6. Immunofluorescence staining of MEPs of the injured biceps brachii and hematoxylin-eosin staining of muscle fibers in the control, blank hydrogel, NEP1-40, and NEPI-40-loaded hydrogel groups at 6 weeks post-surgery. A-D: MEPs of the injured biceps brachii in each group (red) $(n=4$, scale bar $=20 \mu \mathrm{m})$. E-H: Hematoxylin-eosin staining of the biceps brachii in each group $(n=4$, scale bar $=20 \mu \mathrm{m})$. I: The area of MEPs of the biceps brachii on the injured side at 6 weeks post-surgery $(* P<0.05, * * P<0.01)$. J: Muscle fiber diameter of the biceps brachii on the injured side at 6 weeks post-surgery $(* * P<0.01$, ****P $<0.0001)$. 

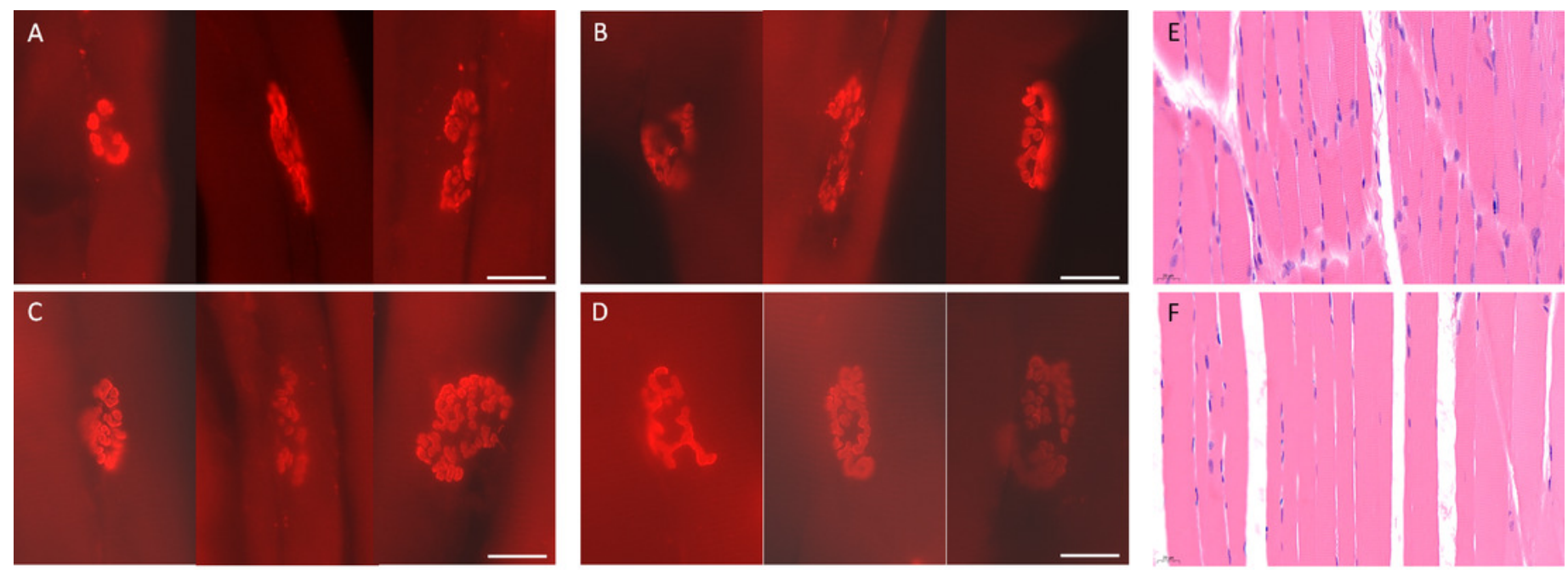

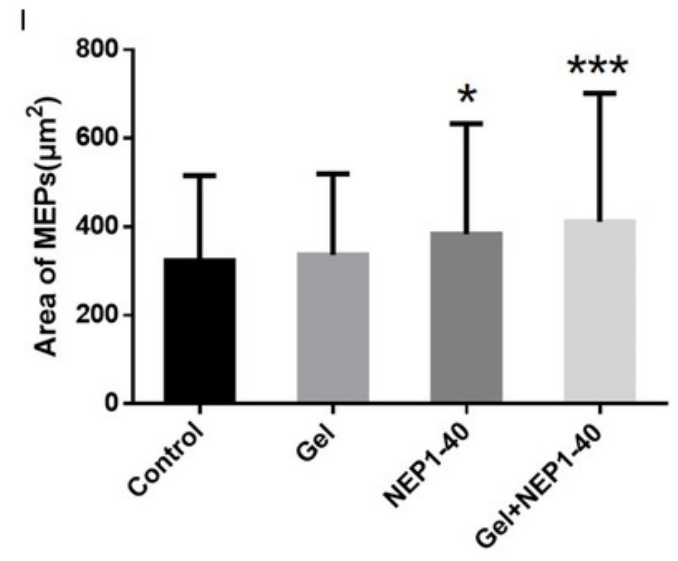

6 weeks after surgery

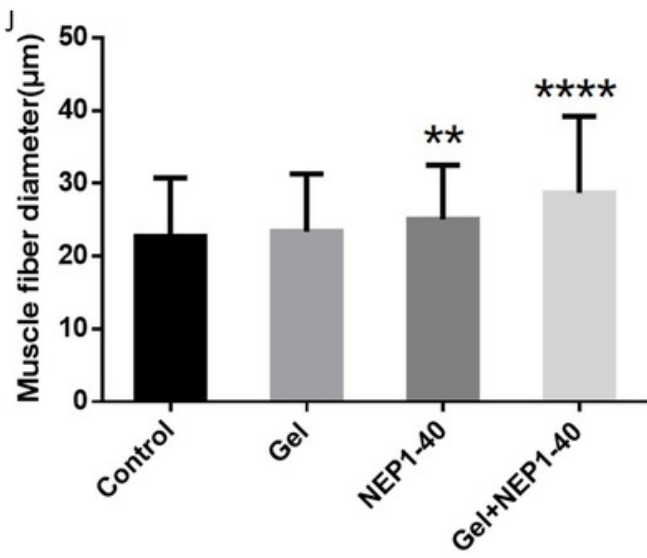

6 weeks after surgery

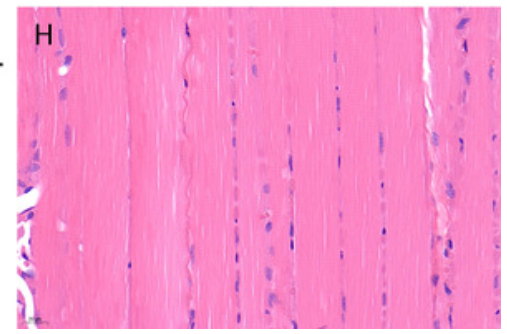


Table $\mathbf{1}$ (on next page)

Grouping and treatments.

Table 1. Grouping and treatments. 
Table 1. Grouping and treatments.

\begin{tabular}{|c|c|c|c|c|}
\hline & Control & Blank & NEP1-40 & NEP1-40-loaded \\
\hline & group & hydrogel group & group & hydrogel group \\
\hline Material loaded & $(-)$ & $100 \mu \mathrm{L}$ & $(-)$ & $100 \mu \mathrm{L}, 0.5 \mathrm{mg} / \mathrm{mL}$ \\
\hline around & & hydrogel & & NEP1-40 -loaded \\
\hline replanted & & & & hydrogel \\
\hline \multicolumn{5}{|l|}{ nerve root } \\
\hline Intraperitoneal & PBS/DMSO & PBS/DMSO & $12.5 \mu \mathrm{g}$ & PBS/DMSO \\
\hline injection & & & NEP1-40 & \\
\hline
\end{tabular}

2 